OPEN ACCESS

Edited by:

Xenia Gonda,

Semmelweis University, Hungary

Reviewed by:

Djamila Bennabi,

University of Franche-Comté, France

Leo Sher,

James J. Peters VA Medical Center,

United States

${ }^{*}$ Correspondence:

Erkki T. Isometsä

erkki.isometsa@hus.fi

Specialty section: This article was submitted to Mood and Anxiety Disorders, a section of the journal

Frontiers in Psychiatry

Received: 06 September 2019 Accepted: 24 March 2020

Published: 17 April 2020

Citation:

Söderholm JJ, Socada JL, Rosenström T. Ekelund $J$ and Isometsä ET (2020) Borderline

Personality Disorder With

Depression Confers Significant

Risk of Suicidal Behavior in

Mood Disorder Patients-

A Comparative Study.

Front. Psychiatry 11:290.

doi: 10.3389/fpsyt.2020.00290

\section{Borderline Personality Disorder With Depression Confers Significant Risk of Suicidal Behavior in Mood Disorder Patients-A Comparative Study}

\author{
John J. Söderholm ${ }^{1}$, J. Lumikukka Socada ${ }^{1}$, Tom Rosenström ${ }^{2,3}$, Jesper Ekelund ${ }^{4}$ \\ and Erkki T. Isometsä ${ }^{1 *}$
}

\begin{abstract}
${ }^{1}$ Department of Psychiatry, University of Helsinki and Helsinki University Central Hospital, Helsinki, Finland, 2 Department of Mental Disorders, Norwegian Institute of Public Health, Oslo, Norway, ${ }^{3}$ Department of Psychology and Logopedics, University of Helsinki, Helsinki, Finland, ${ }^{4}$ Department of Psyhiatry, University of Turku and Turku University Central Hospital, Turku, Finland
\end{abstract}

Objective: We investigated risk factors for suicidal ideation and behavior among currently depressed patients with major depressive disorder (MDD), major depressive episode (MDE) in bipolar disorder (BD), or MDE with comorbid borderline personality disorder (MDE/BPD). We compared current and lifetime prevalence of suicidal ideation and behavior, and investigated dimensional measures of BPD or mixed affective features of the MDE as indicators of risk.

Methods: Based on screening of 1,655 referrals, we recruited 124 psychiatric secondary care outpatients with MDE and stratified them into three subcohorts (MDD, BD, and MDE/ BPD) using the Structured Clinical Interview for DSM-IV I and II. We examined suicidal ideation and behavior with the Columbia Suicide Severity Rating Scale (CSSRS). In addition, we quantified the severity of BPD symptoms and BD mixed features both categorically/diagnostically and dimensionally (using instruments such as the Borderline Personality Disorder Severity Index) in two time frames.

Results: There were highly significant differences between the lifetime prevalences of suicide attempts between the subcohorts, with attempts reported by $16 \%$ of the MDD, $30 \%$ of the BD, and $60 \%$ of the BPD subcohort. Remarkably, the lifetime prevalence of suicide attempts in patients with comorbid BD and BPD exceeded $90 \%$. The severity of BPD features was independently associated with risk of suicide attempts both lifetime and during the current MDE. It also associated in a dose-dependent manner with recent severity of ideation in both BPD and non-BPD patients. In multinominal logistic regression models, hopelessness was the most consistent independent risk factor for severe suicidal ideation in both time frames, whereas younger age and more severe BPD features were most consistently associated with suicide attempts. 
Conclusions: Among patients with major depressive episodes, diagnosis of bipolar disorder, or presence of comorbid borderline personality features both imply remarkably high risk of suicide attempts. Risk factors for suicidal ideation and suicidal acts overlap, but may not be identical. The estimated severity of borderline personality features seems to associate with history of suicidal behavior and current severity of suicidal ideation in dose-dependent fashion among all mood disorder patients. Therefore, reliable assessment of borderline features may advance the evaluation of suicide risk.

Keywords: suicide, suicide attempt, suicidal ideation, major depressive disorder, bipolar disorder, borderline personality disorder, Borderline Personality Disorder Severity Index, Columbia Suicide Severity Rating Scale

\section{INTRODUCTION}

Patients suffering from mood disorders or borderline personality disorder (BPD) are at high risk of suicide. According to psychological autopsy studies, suicide is preceded by psychiatric disorders in the vast majority (c. 90\%) of cases (1, 2). In long-term follow-up studies of patients with bipolar disorder (BD), major depressive disorder (MDD), and BPD, suicide mortality is significant $(3,4)$. Cumulative incidence of suicide among Danish psychiatric patients over 18 years was $4 \%$ for women and $7 \%$ for men with MDD, and 1\% higher in BD (5). The risk of patients with softer BD features, such as periods of elevated mood of subsyndromal intensity or duration, remains unclear (6, 7). Previous suicide attempts (SAs) have been reported in up to half of $\mathrm{BD}$ and a third of MDD patients in psychiatric clinical samples (8). In a prospectively followed cohort of high-risk BPD patients, one third of the patients attempted suicide within 24 months (9). Identifying risk factors for suicidal behavior and ideation in these prevalent psychiatric disorders remains a research priority.

The suicidal process can be conceptualized as potentially progressing from ideation to attempt or death. The risk factors for different stages of suicidal ideation and acts may not be identical (10), and may differ across diagnostic and demographic groups (11), as well as temporally (9). Furthermore, effect of temporally distal risk factors may be mediated by more proximal clinical factors; e.g., influence of childhood maltreatment may be mediated by borderline personality features (12). Therefore, examining putative risk factors across diagnostic groups and stages of the suicidal process is important.

Established risk factors for suicide death in both MDD and BD include male gender and a family history of suicide (13-15), whereas risk factors common for SA include female gender, low age at onset, a recent depressive episode, comorbid anxiety, substance use, cluster B personality disorders, and a family history of suicide $(14,16)$. The risk of SA in mood disorder patients is related in a dose-dependent manner to time spent in depression, most significantly during major depressive episodes (MDE) $(16,17)$. Hopelessness is a risk factor for SA at least in MDD (18). Since BD patients have a lower age at onset and spend more time unwell than MDD patients, their lifetime risk for SA is higher $(8,11)$, and similar mechanisms seem to be at work in BPD (19). In a mixed sample of MDD and BD patients, features suggestive of at least a propensity towards BD were overrepresented among attempters (20). In BPD patients, worsening of comorbid MDD or substance use disorders (SUDs), childhood sexual abuse, traits of aggression and affective dysregulation, psychiatric hospitalization, being of a minority race, or having frequent changes in employment have been prospectively associated with a higher risk of SA (21-24).While BPD per se and certain BPD traits have been noted to be risk factors for SAs in depression, they are rarely dimensionally assessed, and thus, the importance of severity of BPD traits for risk of suicidal ideation and behavior remains unclear.

Although patients with $\mathrm{MDD}, \mathrm{BD}$, and $\mathrm{BPD}$ are at high risk of suicidal behavior, few studies have directly compared the prevalence and risk factors of suicidal ideation and attempts among these patient groups. Methodologically rigorous studies examining the association between adequately quantified BPD symptom/feature severity and suicidal ideation or behavior have been lacking, and the role of softer bipolar features in suicidal ideation and behavior is obscure. Such knowledge may help in identifying the most pertinent risk factors for suicidal behavior and ideation among mood disorder patients, thus helping in targeting treatment and preventive efforts.

The aim of this study was to investigate the prevalence and risk factors of SA among patients with MDE in MDD or BD, with or without comorbid BPD. Our a priori hypotheses were that (a) lifetime and recent SA are more prevalent and suicidal ideation more severe among BD and BPD/MDD patients than among MDD patients, (b) severe features or symptoms of BPD is a risk factor for suicidal ideation and behavior, and (c) mixed symptoms during depressive episodes are correlated with risk of SA or suicidal ideation.

\section{MATERIALS AND METHODS}

This was an observational cohort study conducted in Helsinki, Finland. Our sampling frame consisted of all patients referred with a possible diagnosis of affective disorder to the Northern and Eastern psychiatric outpatient clinics (offering secondary psychiatric care to a population of 234,415 adults).

The study was conducted according to the tenets of the Declaration of Helsinki (2013). Written informed consent was 
obtained from each participant. The research protocol was approved by the Ethics Committee of the Helsinki and Uusimaa Hospital District, and the research permit was granted by the City of Helsinki.

\section{Inclusion and Exclusion Criteria}

Inclusion criteria were (1) current MDE, (2) a MontgomeryÅsberg Depression Rating Score (MADRS) $\geq 15$, and (3) age 1850 years. Exclusion criteria were (1) acute psychotic symptoms, (2) a diagnosis of schizophrenia or another primary psychotic disorder (not including MDD or BD with previous psychotic symptoms), (3) substance dependence (as in DSM-IV) with any current use, (4) ongoing use of large quantities of alcohol (24 units/week for men and 16 units/week for women) or benzodiazepine-type drugs $\geq 15 \mathrm{mg}$ /day diazepam equivalents) in the last month, (5) any use of illicit drugs in the last month other than sporadic use of cannabis (once monthly in the last three months), (6) schizotypal or antisocial personality disorder, (7) a major neurocognitive or neurodevelopmental disorder, and (8) insufficient proficiency in the Finnish language to participate.

\section{Stratification and Recruitment}

The patients were stratified into three principal diagnosis subcohorts: (1) MDD (no BPD), (2) BD, and (3) BPD with $\mathrm{MDE}$. In case of comorbid $\mathrm{BD}$ and $\mathrm{BPD}$, all $\mathrm{BD}$ type I patients were assigned to the $\mathrm{BD}$ subcohort, otherwise subcohort assignment was by principal clinical diagnosis.

Two of the authors (LS and JS) screened a total of 1,655 referrals for subjects. We preliminarily stratified these into three recruitment pools according to likely clinical diagnosis: (1) MDD with current MDE, (2) probable MDE in $\mathrm{BD}$, and (3) probable MDE with comorbid BPD, and by gender. We aimed for equal representation in the strata, enriching currently underrepresented categories. If necessary, the patient to be interviewed was randomly selected within the stratum. Of screened referrals, 436 did not meet inclusion criteria, 855 were not contacted due to limited capacity or not reached, and 209 declined to participate. Thirty-one patients were excluded after a clinical interview, leaving a sample of 124 subjects. These were classified into the three final subcohorts according to final diagnosis.

Altogether, 31 patients were included in the BPD subcohort, 43 in the BD subcohort, and the remaining 50 in the MDD subcohort. Of these, 14 patients met the DSM criteria for both $\mathrm{BD}$ (type II) and BPD, eight of these were included in the BPD and six in the $\mathrm{BD}$ subcohort. Fourteen patients (six from the $\mathrm{BPD}$, three from the $\mathrm{BD}$, and five from the MDD subcohort) did not complete the Columbia Suicide Severity Rating Scale (CSSRS) (25) and are not included in the analyses of suicidal ideation and behavior. These patients did not differ from the main sample in MADRS scores or reported current suicidal thoughts or actions in the SCID.

\section{Interviews, Questionnaires, and Inter- Rater Reliability}

All subjects were interviewed using the Structured Clinical Interview for DSM-IV-TR (SCID-I) (26) and SCID-II (27) (with the following personality disorders: paranoid, schizotypal, borderline, histrionic, narcissistic, avoidant, dependent, and obsessive-compulsive), MADRS (28), the Young Mania Rating Scale (YMRS) (29), Borderline Personality Disorder Severity Index (BPDSI) (30), part of the bipolar specifier (31), the Social and Occupational Functioning Assessment Scale (SOFAS) (32), and the CSSRS (25) in the use of which the performing authors (LS and JS) had received extensive training. Subjects were asked to complete questionnaires, including the Beck Depression Inventory-II (BDI-II) (33), the Patient Health Questionnaire-9 (PHQ-9) (34), the Overall Anxiety Severity and Impairment Scale (OASIS) (35), the McLean Screening Instrument for BPD (MSI-BPD) (36), the Perceived Social Support Scale (PSSS) (37), and the Beck Hopelessness Scale (HS) (38). We quantified possible mixed features of the index MDE via interview, scoring the occurrence of manic symptoms during the current MDE from 0 to 5 (Mix-MDE, see Supplementary Material for details). The subjects were met three times. Interrater reliability for principal diagnoses, tested by rating of videotaped interviews, was excellent (Cohen's $\kappa$ for MDD 1.0, for bipolar disorder 0.898, for BPD 0.891).

As per the C-SSRS, we explored the severity of the subjects' most severe lifetime and recent suicidal ideation. This was quantified on the Suicidal Ideation Scale (SIS). Suicidal behavior included preparative acts in addition to aborted and interrupted and completed attempts (25). We classified our subjects into the following categories for both most severe lifetime and recent suicidali ideation: (1) no or mild suicidal ideation (SIS 0-2); (2) severe ideation (SIS 3-5) without SA, and (3) SA. For analyzing bipolarity dimensionally, we classified patients into the following ordinal categories: (1) no evidence of bipolarity, (2) bipolarity specifier positive but DSM-5 negative (subsyndromal hypomanias with a duration of 1-3 days only) (6, 7), (3) DSM-5 BD type II, and (4) DSM-5 BD type I. This ordinal measure was then used as a covariate in statistical analyses.

\section{Statistical Analyses}

Data were assembled into a database using SQLite (http://www. sqlite.org/) and analyzed with IBM SPSS Statistics (https://www. ibm.com/analytics/us/en/technology/spss/) version 24. Chisquare test, Fisher's exact test, t-tests, and ANOVA were used in group comparisons. Furthermore, we analyzed correlates of suicidal ideation and behavior using multinomial logistic regression (using the latest available symptom severity scores), while subtracting suicide-related items from the MADRS, from the BPDSI, and from the number of positive BPD criteria in the SCID-II to avoid circularity.

Several multinomial logistic regression models were compared in assessing risk factors of lifetime and recent significant suicidal ideation and SA. Because each covariate has multiple regression coefficients in the multinomial model, overall significance of a covariate is tested by comparing models with and without it using a likelihood-ratio test. The covariates used in the initial lifetime model were gender, age, diagnosis of SUD, eating disorder, the bipolarity ordinal measure, most recent MADRS, HS, OASIS, number of positive BPD criteria (except suicidal ideation or behavior) in the SCID-II, PSSS, and duration 
of illness. In the initial model for recent suicidal ideation and behavior, the factors used were gender and diagnoses of eating or substance use disorders, while the covariates were the bipolarity ordinal measure, age, HS, OASIS, MADRS, BPDSI, Mix-MDE, PSSS, and duration of illness. Variables that were neither theoretically central nor statistically significant (i.e., for the lifetime model diagnosis of eating disorder or SUD, OASIS, PSSS and duration of illness; for the recent model diagnosis of eating disorder or SUD, OASIS, PSSS, duration of illness), were excluded from our final model.

\section{RESULTS}

No significant differences existed between the three patient subcohorts in gender, age, severity of depression, hopelessness, or level of functioning or perceived social support (Table 1). The severity of borderline symptoms was expectedly significantly higher in the BPD subcohort, and the YMRS scores lower in the MDD cohort, than in the others. Most (79\%) of the patients in all subcohorts were treated with pharmacotherapy. However, they differed in use of mood stabilizers, used by $4 \%, 26 \%$, and $10 \%$; antipsychotics, used by $10 \%, 30 \%$, and $16 \%$; and antidepressants, used by $74 \%$ of the MDD, $56 \%$ of the BD, and $87 \%$ of the BPD subcohorts, respectively.

Prevalence and severity of suicidal behavior and ideation is reported in Table 2. There were highly significant differences ( $p<0.001)$ between the subcohorts in the lifetime prevalences of suicide attempts, suicidal behavior, and non-suicidal self-injury, all of which were most common in the BPD subcohort (in which they were reported by the majority of subjects). The median SIS for most severe lifetime suicidal ideation was 4 in the BPD subcohort and 3 in the others, and there were also significant differences between the subcohorts in the lifetime prevalence of significant suicidal ideation $(\mathrm{p}=0.001)$, whereas the intersubcohort differences in the prevalence of any suicidal ideation did not reach statistical significance $(p=0.066)$. During the preceding month the corresponding median SIS scores were 4 (the BPD subcohort) and 1 (others).

When grouping patients purely into four groups by $\mathrm{MDD} / \mathrm{BD}$ and BPD-diagnosis status (instead of by subcohorts) there were significant differences in the lifetime prevalences of SA (Fisher's exact test, $\mathrm{p}<0.001$ ), with earlier SA reported by $15.6 \%$ of MDD patients without comorbid BPD, $25.0 \%$ of BD patients without BPD, $44.4 \%$ of MDD patients with comorbid BPD, and $90.9 \%$ (10 out of 11) among BD patients with comorbid BPD (Figure 1).

The multinominal regression model for lifetime suicidal behavior and ideation explained these phenomena with high statistical significance $(2 \mathrm{LL}=184.013 ; \chi 2(12)=42.635 ; \mathrm{p}<$ 0.001 ), and a moderate effect size (Nagelkerke pseudo-R2 = 0.368 , Table 3 ). The covariates that significantly contributed to the model were age $(\mathrm{p}=0.040)$, MADRS $(\mathrm{p}=0.003)$, HS $(\mathrm{p}=$ $0.019)$, and number of positive BPD criteria $(\mathrm{p}=0.004)$, but not the bipolarity measure $(\mathrm{p}=0.346)$ or $\operatorname{gender}(\mathrm{p}=0.151)$.

The recent suicidal behavior and ideation model was also highly significant $(-2 L L=149.186 ; \chi 2(14)=44.670 ; \mathrm{p}<0.001)$, and the effect size moderate (Nagelkerke pseudo-R2 $=0.403$, Table 4). Age $(\mathrm{p}=0.009)$, HS $(\mathrm{p}=0.010)$, MADRS $(\mathrm{p}=0.013)$, and BPDSI $(\mathrm{p}<0.001)$ were significant contributors to this overall model, while gender $(\mathrm{p}=0.552)$, the bipolarity ordinal measure $(\mathrm{p}=0.984)$, and Mix-MDE $(\mathrm{p}=0.502)$ were not.

TABLE 1 | Sociodemographic and clinical characteristics by subcohort.

\begin{tabular}{|c|c|c|c|c|c|c|c|c|c|c|c|c|c|c|c|}
\hline & & & \multicolumn{12}{|c|}{ Subcohort } & \multirow[b]{3}{*}{ Sig. } \\
\hline & & & \multicolumn{3}{|c|}{ MDD } & \multicolumn{3}{|c|}{ Bipolar Disorder } & \multicolumn{3}{|c|}{ BPD } & \multicolumn{3}{|c|}{ Total } & \\
\hline & & & Count & $\% /$ Mean & SD & Count & $\% /$ Mean & SD & Count & $\% /$ Mean & SD & Count & $\% /$ Mean & SD & \\
\hline \multirow[t]{2}{*}{ Sex } & Male & & 21 & $42.0 \%$ & & 12 & $27.9 \%$ & & 8 & $25.8 \%$ & & 41 & $33.1 \%$ & & $0.237^{a)}$ \\
\hline & Female & & 29 & $58.0 \%$ & & 31 & $72.1 \%$ & & 23 & $74.2 \%$ & & 83 & $66.9 \%$ & & \\
\hline Age & & & & 31.4 & 10.2 & & 31.6 & 9.1 & & 28.5 & 7.6 & & 30.8 & 9.2 & $0.301^{\mathrm{b})}$ \\
\hline \multirow[t]{3}{*}{ Bipolar Disorder } & Total & & 0 & $0.0 \%$ & & 43 & $100.0 \%$ & & 8 & $25.8 \%$ & & 51 & $41.1 \%$ & & $<0.001^{\mathrm{a})}$ \\
\hline & & Type ॥ & 0 & $0.0 \%$ & & 33 & $76.7 \%$ & & 8 & $25.8 \%$ & & 41 & $33.1 \%$ & & \\
\hline & & Type I & 0 & $0.0 \%$ & & 10 & $23.3 \%$ & & 0 & $0.0 \%$ & & 10 & $8.1 \%$ & & \\
\hline BPD & & & 0 & $0.0 \%$ & & 6 & $14.0 \%$ & & 31 & $100.0 \%$ & & 37 & $29.8 \%$ & & $<0.001^{\text {a) }}$ \\
\hline SUD & & & 4 & $8.0 \%$ & & 19 & $44.2 \%$ & & 14 & $45.2 \%$ & & 37 & $29.8 \%$ & & $<0.001^{\mathrm{a})}$ \\
\hline Anxiety disorder & & & 37 & $74.0 \%$ & & 34 & $79.1 \%$ & & 29 & $93.5 \%$ & & 100 & $80.6 \%$ & & $0.095^{a)}$ \\
\hline Eating disorder & & & 10 & $20.0 \%$ & & 13 & $30.2 \%$ & & 10 & $32.3 \%$ & & 33 & $26.6 \%$ & & $0.405^{a)}$ \\
\hline MADRS & & & & 25.0 & 6.0 & & 25.2 & 6.9 & & 26.3 & 6.0 & & 25.4 & 6.3 & $0.639^{b)}$ \\
\hline BDI-II & & & & 28.7 & 10.1 & & 30.6 & 10.7 & & 33.6 & 9.8 & & 30.6 & 10.3 & $0.166^{b)}$ \\
\hline YMRS & & & & 1.7 & 2.7 & & 3.4 & 3.7 & & 3.5 & 2.6 & & 2.8 & 3.2 & $0.007^{b)}$ \\
\hline OASIS & & & & 11.8 & 3.1 & & 12.6 & 3.7 & & 12.9 & 4.6 & & 12.3 & 3.7 & $0.419^{b)}$ \\
\hline $\mathrm{HS}$ & & & & 12.5 & 3.9 & & 11.8 & 4.8 & & 10.9 & 3.9 & & 11.9 & 4.2 & $0.287^{b)}$ \\
\hline PSSS & & & & 40.0 & 13.1 & & 45.0 & 11.6 & & 40.0 & 11.6 & & 42.0 & 12.4 & $0.154^{b)}$ \\
\hline SOFAS & & & & 56.2 & 11.6 & & 57.4 & 13.0 & & 60.0 & 10.7 & & 57.5 & 11.9 & $0.446^{b)}$ \\
\hline BPDSI & & & & 14.4 & 8.3 & & 16.8 & 6.6 & & 20.8 & 13.8 & & 16.8 & 9.7 & $0.013^{b)}$ \\
\hline
\end{tabular}

a) Pearson $\chi^{2}$.

b) One way ANOVA.

BDI2, Beck depression inventory 2; BPD, Borderline personality disorder; BPDSI, Borderline personality disorder severity index; HS, Hopelessness scale; MADRS, Montgomery Åsberg Depression Rating Scale; MDD, Major depressive disorder; PSSS, Perceived social support scale; SOFAS, Social and occupational functioning assessment scale; SUD, Substance use disorder; YMRS, Young mania rating scale. 
TABLE 2 | Prevalence and severity of suicidal behavior and ideation.

\begin{tabular}{|c|c|c|c|c|c|c|c|c|c|c|c|c|c|c|}
\hline & & \multicolumn{12}{|c|}{ Subcohort } & \multirow[b]{3}{*}{ Sig. } \\
\hline & & \multicolumn{3}{|c|}{ MDD } & \multicolumn{3}{|c|}{ Bipolar Disorder } & \multicolumn{3}{|c|}{ BPD } & \multicolumn{3}{|c|}{ Total } & \\
\hline & & Count & $\% /$ Mean & SD & Count & $\% /$ Mean & SD & Count & $\% /$ Mean & SD & Count & $\% /$ Mean & SD & \\
\hline \multirow[t]{7}{*}{ Lifetime } & Attempts & 7 & $15.6 \%$ & - & 12 & $30.0 \%$ & - & 15 & $60.0 \%$ & - & 34 & $30.9 \%$ & - & $<0.001^{\mathrm{a})}$ \\
\hline & Behaviour & 12 & $26.7 \%$ & - & 16 & $40.0 \%$ & - & 20 & $80.0 \%$ & - & 48 & $43.6 \%$ & - & $<0.001^{\mathrm{a})}$ \\
\hline & Non-suicidal self injury & 6 & $22.5 \%$ & - & 9 & $22.5 \%$ & - & 16 & $64.0 \%$ & - & 31 & $28.2 \%$ & - & $<0.001^{\text {a) }}$ \\
\hline & Ideation & 39 & $86.7 \%$ & - & 32 & $80.0 \%$ & - & 25 & $100.0 \%$ & - & 96 & $87.3 \%$ & - & $0.066^{a)}$ \\
\hline & Significant ideation & 31 & $68.9 \%$ & - & 24 & $60.0 \%$ & - & 25 & $100.0 \%$ & - & 80 & $72.7 \%$ & - & $0.001^{a)}$ \\
\hline & SIS & & 3.00 & 1.68 & & 2.68 & 1.86 & & 4.08 & 0.76 & & 3.13 & 1.67 & $0.003^{b)}$ \\
\hline & SIIR & & 13.02 & 17.07 & & 12.28 & 7.19 & & 15.84 & 3.00 & & 13.39 & 6.52 & $0.088^{b)}$ \\
\hline \multirow[t]{7}{*}{ Recent } & Attempts & 3 & $6.7 \%$ & - & 2 & $5.0 \%$ & - & 5 & $20.0 \%$ & - & 10 & $9.1 \%$ & - & $0.138^{\mathrm{c})}$ \\
\hline & Behaviour & 8 & $17.8 \%$ & - & 4 & $10.0 \%$ & - & 8 & $32.0 \%$ & - & 20 & $18.2 \%$ & - & $0.097^{\mathrm{c})}$ \\
\hline & Non-suicidal self injury & 3 & $6.7 \%$ & - & 6 & $15.0 \%$ & - & 9 & $36.0 \%$ & - & 18 & $16.4 \%$ & - & $0.008^{c)}$ \\
\hline & Ideation & 29 & $64.4 \%$ & - & 21 & $52.5 \%$ & - & 18 & $72.0 \%$ & - & 68 & $61.8 \%$ & - & $0.278^{\mathrm{a})}$ \\
\hline & Significant ideation & 16 & $35.6 \%$ & - & 11 & $27.5 \%$ & - & 13 & $52.0 \%$ & - & 40 & $36.4 \%$ & - & $0.124^{a)}$ \\
\hline & SIS & & 1.76 & 1.80 & & 1.25 & 1.48 & & 2.04 & 1.65 & & 1.64 & 1.67 & $0.147^{\mathrm{b})}$ \\
\hline & SIIR & & 8.13 & 7.20 & & 6.90 & 7.08 & & 9.08 & 5.97 & & 7.90 & 6.89 & $0.447^{\mathrm{b})}$ \\
\hline
\end{tabular}

a) Pearson $\chi^{2}$.

b) One-Way ANOVA.

${ }^{c)}$ Fisher's exact test.

BPD, Borderline personality disorder; MDD, Major depressive disorder; SIS, Suicidal ideation scale; SIIR, Suicidal ideation intensity rating.

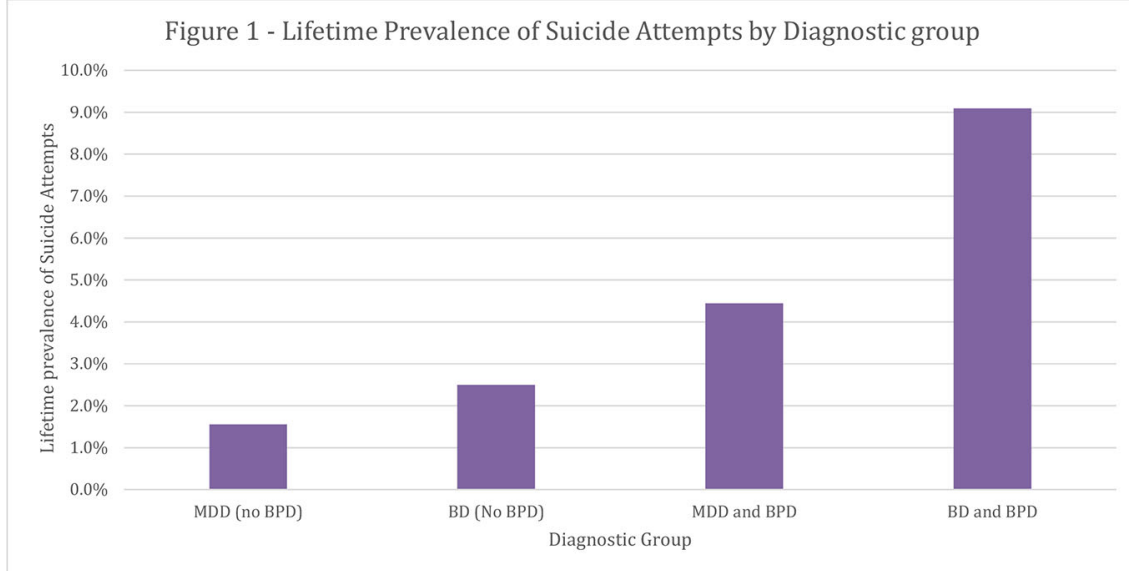

FIGURE 1 | Lifetime prevalence of suicide attempts by diagnostic group.

A significant correlation existed between severity of recent suicidal ideation and BPDSI scores (Pearson correlation: 0.383, $\mathrm{p}<0.001)$, which remained significant both among BPD (Pearson correlation: 0.586, $\mathrm{p}<0.001$ ) and non-BPD patients (Pearson correlation: 0.278, $\mathrm{p}=0.012$ ).

As a post-hoc analysis, we replaced the total BPDSI score in the multinomial regression model for recent suicidal behavior and ideation with the BPDSI subscores (except the self-injury category). The model was significant ( $p>0.001)$. Of the BPDSI subcategories, sensitivity to abandonment $(\mathrm{p}=0.004, \mathrm{OR}=$ $2.070,95 \%$ CI [1.264 - 3.391]) and anger/rage conferred increased odds of having made an attempt $(\mathrm{p}=0.007$, OR $=$ 2.719, 95\% CI [1.318 - 5.611]); the others did not reach statistical significance.

\section{DISCUSSION}

In this observational clinical study of treatment-seeking major depressive patients, we investigated the prevalence of and risk factors for suicidal ideation and attempts. We compared depressed patients with $\mathrm{MDD}, \mathrm{BD}$, and/or BPD. As hypothesized, we found marked differences in the lifetime prevalence of suicide attempts, which was twofold in the $\mathrm{BD}$ and fourfold in the BPD/MDE subcohort compared with MDD patients. Severity of BPD features was also strongly associated with suicidal ideation, both recently and over the lifetime. However, we did not find mixed symptoms during the index MDE to be a risk factor for suicidal behavior and ideation in this cross-sectional and retrospective analysis. 
TABLE 3 | Most severe lifetime suicidal ideation/behavior - Multinominal logistic regression models.

\begin{tabular}{|c|c|c|c|c|c|c|c|c|c|c|c|}
\hline \multicolumn{2}{|l|}{ Suicidality $^{\text {a) }}$} & \multicolumn{5}{|c|}{ Uncontrolled } & \multicolumn{5}{|c|}{ Multiple (adjusted) regression } \\
\hline & & \multirow{2}{*}{$\begin{array}{c}\text { B } \\
-0.032\end{array}$} & \multirow{2}{*}{$\begin{array}{l}\mathbf{O R}^{\mathbf{b})} \\
0.969\end{array}$} & \multicolumn{2}{|c|}{$95 \% \mathrm{Cl}$} & \multirow{2}{*}{$\begin{array}{c}\text { Sig. } \\
0.291\end{array}$} & \multirow{2}{*}{$\begin{array}{c}\text { B } \\
-0.043\end{array}$} & \multirow{2}{*}{$\begin{array}{l}\mathbf{O R}^{\mathbf{b})} \\
0.957\end{array}$} & \multicolumn{2}{|c|}{$95 \% \mathrm{Cl}$} & \multirow{2}{*}{$\frac{\text { Sig. }}{0.234}$} \\
\hline Significant ideation, no attempt & Age & & & 0.914 & 1.027 & & & & 0.891 & 1.028 & \\
\hline & Bipolarity Ordinal & -0.360 & 0.698 & 0.419 & 1.161 & 0.166 & -0.399 & 0.671 & 0.389 & 1.157 & 0.151 \\
\hline & MADRS & -0.008 & 0.992 & 0.894 & 1.100 & 0.874 & 0.043 & 1.044 & 0.932 & 1.170 & 0.455 \\
\hline & HS & 0.105 & 1.111 & 0.987 & 1.249 & 0.080 & 0.140 & 1.150 & 1.002 & 1.321 & 0.047 \\
\hline & $\mathrm{Nr}$ of pos. BPD criteria & 0.045 & 1.046 & 0.974 & 1.124 & 0.218 & 0.268 & 1.307 & 0.943 & 1.813 & 0.108 \\
\hline & Sex (male) & 1.190 & 3.286 & 1.025 & 10.528 & 0.045 & 1.223 & 3.396 & 0.957 & 12.051 & 0.059 \\
\hline \multirow[t]{6}{*}{ Attempt } & Age & -0.052 & 0.949 & 0.903 & 0.997 & 0.016 & -0.081 & 0.922 & 0.864 & 0.985 & 0.018 \\
\hline & Bipolarity Ordinal & -0.137 & 0.872 & 0.584 & 1.303 & 0.396 & -0.213 & 0.808 & 0.495 & 1.321 & 0.443 \\
\hline & MADRS & 0.090 & 1.094 & 1.005 & 1.192 & 0.003 & 0.154 & 1.166 & 1.053 & 1.291 & 0.003 \\
\hline & HS & 0.125 & 1.133 & 1.027 & 1.252 & 0.009 & 0.170 & 1.185 & 1.044 & 1.345 & 0.009 \\
\hline & Nr of pos. BPD criteria & 0.080 & 1.083 & 1.018 & 1.152 & 0.003 & 0.461 & 1.586 & 1.172 & 2.146 & 0.001 \\
\hline & Sex (male) & 0.602 & 1.825 & 0.667 & 4.998 & 0.201 & 0.786 & 2.194 & 0.658 & 7.312 & 0.190 \\
\hline
\end{tabular}

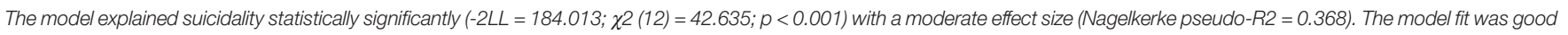
and resulted in statistically similar predicted and observed suicidality distributions (Pearson and deviance statistic yielded $p=0.185$ and $p=0.862$, respectively).

a) The reference category is: No or mild ideation (SIS 0-2).

b) OR equals $\operatorname{Exp}(B)$.

BPD, Borderline personality disorder; HS, Hopelessness scale; MADRS, Montogomery Åsberg Depression Rating Scale; OR, Odds ratio; SIS, Suicidal ideation scale.

TABLE 4 | Recent suicidal idation/behavior - multinominal logistic regression models.

\begin{tabular}{|c|c|c|c|c|c|c|c|c|c|c|c|}
\hline \multirow[b]{3}{*}{ Significant ideation, no attempt } & \multirow[b]{3}{*}{ Bipolarity Ordinal } & \multicolumn{5}{|c|}{ Uncontrolled } & \multicolumn{5}{|c|}{ Controlled } \\
\hline & & \multirow{2}{*}{$\begin{array}{c}\text { B } \\
-0.076\end{array}$} & \multirow{2}{*}{$\begin{array}{c}\text { OR }^{\mathbf{b})} \\
0.927\end{array}$} & \multicolumn{2}{|c|}{$95 \% \mathrm{Cl}$} & \multirow{2}{*}{$\begin{array}{l}\text { Sig. } \\
0.697\end{array}$} & \multirow{2}{*}{$\begin{array}{c}\text { B } \\
0.045\end{array}$} & \multirow{2}{*}{$\frac{\mathbf{O R}^{\mathbf{b})}}{1.046}$} & \multicolumn{2}{|c|}{$95 \% \mathrm{Cl}$} & \multirow{2}{*}{$\frac{\text { Sig. }}{0.856}$} \\
\hline & & & & 0.631 & 1.360 & & & & 0.643 & 1.701 & \\
\hline & Age & -0.029 & 0.971 & 0.926 & 1.018 & 0.226 & -0.082 & 0.921 & 0.862 & 0.984 & 0.015 \\
\hline & HS & 0.137 & 1.147 & 1.038 & 1.267 & 0.007 & 0.181 & 1.199 & 1.058 & 1.358 & 0.004 \\
\hline & MADRS & 0.130 & 1.139 & 1.051 & 1.235 & 0.002 & 0.135 & 1.145 & 1.038 & 1.263 & 0.007 \\
\hline & BPDSI & 0.103 & 1.108 & 1.044 & 1.177 & 0.001 & 0.134 & 1.144 & 1.057 & 1.237 & 0.001 \\
\hline & Mix-MDE & 0.002 & 1.002 & 0.945 & 1.062 & 0.950 & -0.050 & 0.951 & 0.869 & 1.041 & 0.274 \\
\hline & Male sex & 0.218 & 1.243 & 0.525 & 2.942 & 0.620 & 0.612 & 1.844 & 0.633 & 5.375 & 0.262 \\
\hline \multirow[t]{7}{*}{ Attempt } & Bipolarity Ordinal & -0.110 & 0.896 & 0.482 & 1.663 & 0.727 & 0.026 & 1.026 & 0.486 & 2.167 & 0.947 \\
\hline & Age & -0.086 & 0.918 & 0.836 & 1.008 & 0.073 & -0.112 & 0.894 & 0.804 & 0.994 & 0.039 \\
\hline & HS & 0.010 & 1.010 & 0.877 & 1.164 & 0.885 & 0.090 & 1.094 & 0.928 & 1.290 & 0.286 \\
\hline & MADRS & 0.089 & 1.093 & 0.970 & 1.232 & 0.143 & 0.110 & 1.116 & 0.979 & 1.274 & 0.101 \\
\hline & BPDSI & 0.125 & 1.133 & 1.038 & 1.237 & 0.005 & 0.136 & 1.146 & 1.029 & 1.277 & 0.013 \\
\hline & Mix-MDE & 0.029 & 1.029 & 0.945 & 1.121 & 0.507 & -0.042 & 0.959 & 0.846 & 1.087 & 0.514 \\
\hline & Male sex & -0.199 & 0.820 & 0.194 & 3.472 & 0.787 & 0.174 & 1.190 & 0.241 & 5.870 & 0.831 \\
\hline
\end{tabular}


and resulted in statistically similar predicted and observed suicidality distributions(Pearson $p=0.324$, deviance $p=0.999$ ).

a) The reference category is: No or mild ideation (SIS 0-2).

${ }^{b)} \mathrm{OR}$ equals $\operatorname{Exp}(B)$.

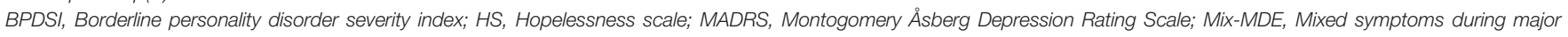
depressive episode; OR, Odds ratio; SIS, Suicidal ideation scale.

\section{Strengths and Weaknesses}

Strengths of this study include the comparison of three clinically and epidemiologically central diagnostic groups with a high suicide risk, the representativeness of recruitment by stratified sampling, the standardized and rigorous assessment of dimensional aspects of psychiatric pathology such as BPD and suicidal ideation and behavior with the BPDSI and the CSSRS, the excellent inter-rater reliability, and the analysis of our results in two time frames (current and lifetime). Our main limitations include moderate sample size, retrospective evaluations, and unknown inter-rater reliability of the BPDSI. Features of our study relevant for interpretation and generalizability of the findings include the observational design, i.e., treatments provided were not controlled by the researchers, age range $18-50$ years, and deliberate focus on proximal, rather than temporally distal, risk factors.

\section{Suicidal Behavior}

We found marked differences in suicidal behavior and ideation of the patient groups. Approximately one-third of patients reported an earlier SA, which is in line with earlier Finnish studies of patients with mood disorders (39). The proportion of patients in the MDD subcohort reporting a history of earlier SA was $15 \%$, comparable with the findings of STAR ${ }^{\star} \mathrm{D}$ and iSpot-D $(40,41)$ as well as with other Finnish studies (42). Relative to the MDD subcohort, in the 
BD subcohort previous SAs were twice as prevalent (30\%; slightly lower than previous reports of around $40 \%)(43,44)$ and in the BPD subcohort four times more prevalent (60\%). Remarkably, over $90 \%$ of $\mathrm{BD}$ patients with comorbid $\mathrm{BPD}$ reported a previous SA, which may indicate cumulative risk, although this finding is in need of replication. Suicide attempts during the index MDE were reported by approximately $9 \%$ of patients, which is somewhat lower than in previous Finnish studies of MDD (42) and BD patients, which however, included inpatients. The fact that lifetime, but not recent, prevalence of SA was higher in the BD than in the MDD subcohort is in line with earlier evidence that the increased SA risk of $\mathrm{BD}$ patients (relative to MDD patients) is due to their spending more time unwell. Our findings suggest that BD patients have a twofold higher risk of SA than MDD patients, MDD/BPD patients have a twofold higher risk than $\mathrm{BD}$ patients, and the risk is cumulative in $\mathrm{BD} / \mathrm{BPD}$ patients, with the risk being six-fold higher than in MDD patients without BPD.

\section{Suicidal Ideation}

Suicidal ideation is a multifaceted concept, and how much of it is found in a particular sample depends on both how the concept is defined and the tools with which it is measured (45). The CSSRS, which is aimed to capture a wide range of suicidal ideation in a precise manner, indicated some degree of suicidal ideation in the vast majority $(87.3 \%)$ of our sample. This is a larger proportion than in many previous studies (39) probably indicating a greater sensitivity of the instrument used, although our sampling procedure, which aimed to include sufficient numbers of BPD patients, may also have contributed. Recent suicidal ideation was reported by approximately $60 \%$ of $\mathrm{BD}$ and $\mathrm{MDD}$ patients, comparable to earlier BD (44) and MDD studies (42) As well as being more common, suicidal ideation was also more severe in the BPD subcohort; the median patient in the BPD group reported having had suicidal thoughts with specific methods and some intent to act, whereas median patients in the other subcohorts did not report ever having had suicidal intent. Our findings indicate that $\mathrm{BPD} / \mathrm{MDE}$ patients have a propensity towards more severe suicidal ideation than other mood disorder patients, and that having had suicidal intent is common in this patient group.

\section{Risk Factors and Correlations}

We examined risk factors for suicidal ideation and attempts, and expectedly found that hopelessness was independently correlated with lifetime risk of previous attempts and severe suicidal ideation, whereas lower age, increased severity of depressive symptoms, and features of BPD were correlated with a higher risk of previous SA. A younger age and more severe features of BPD were independently correlated with a higher risk of both recent attempts and severe ideation, whereas hopelessness and severity of depressive symptoms were related only to the risk of having severe suicidal ideation.

We found that severity of BPD features was independently correlated with suicidal ideation and acts and related to recent severity of suicidal ideation in a dose-dependent manner. To our knowledge, this is the first study to analyze the role of current severity of BPD features, reliably measured by the BPDSI, as a risk factor for suicidal behavior and ideation. According to post-hoc analyses, sensitivity to abandonment, feelings of emptiness, and outbursts of anger or rage may be significantly correlated with significant suicidal ideation or behavior.

In contrast to our hypotheses, we could not confirm earlier reports of a correlation between bipolar mixed states and either suicidal ideation or behavior. Some reasons for this may be our inclusion criterion of current MDE, which may have selected against mixed states, and the lack of precise information regarding the affective states of patients at the time of previous attempts.

\section{Conclusions}

In conclusion, we found marked differences in the prevalence and severity of suicidal ideation and behavior between depressed patients with MDD, BD, or BPD with MDD. The lifetime prevalence of suicidal attempts and other behavior was higher in the $\mathrm{BD}$ than in the MDD subcohort, but highest in the BPD/ MDD subcohort. BD with comorbid BPD may confer an additive and thus remarkably high risk of suicide attempts. Of all risk factors examined, dimensional measures of BPD severity were most consistently and significantly correlated with the risk for significant ideation and recent SAs. In the light of this study, severity of BPD features is a central modifiable $(46,47)$ risk factor for suicidal behavior and ideation among mood disorder patients. Thus, measuring this severity reliably (e.g., with the BPDSI) should be implemented in future suicidological research. Clinically, assessment of borderline features may advance evaluation of suicide risk, and their treatment help suicide prevention.

\section{DATA AVAILABILITY STATEMENT}

The datasets for this manuscript are not publicly available, because the research permit of the study does not allow it. Requests to access the datasets should be directed to EI (erkki.isometsa@hus.fi).

\section{ETHICS STATEMENT}

The studies involving human participants were reviewed and approved by the Ethics Committee of the Helsinki and Uusimaa Hospital District. The patients/participants provided their written informed consent to participate in this study.

\section{AUTHOR CONTRIBUTIONS}

JJS, JLS, TR, JE, and EI are part of the research team. EI, JE, JJS, and JLS designed the study. JLS and JJS recruited, interviewed, and assessed the patients. JJS undertook the analysis of this manuscript with TR acting as statistical expert. JJS wrote the first draft of the manuscript. All authors discussed the results and implications and commented on the manuscript. All authors have contributed to and approved the final manuscript. 


\section{FUNDING}

This study has been funded by research grants from the City of Helsinki, the Helsinki and Uusimaa Hospital District, and the Finnish Psychiatric Association.

\section{REFERENCES}

1. Cavanagh JT, Carson AJ, Sharpe M, Lawrie SM. Psychological autopsy studies of suicide: a systematic review. Psychol Med (2003) 33(3):395-405. doi: 10.1017/S0033291702006943

2. Henriksson MM, Aro HM, Marttunen MJ, Heikkinen ME, Isometsa ET, Kuoppasalmi KI, et al. Mental disorders and comorbidity in suicide. Am J Psychiatry (1993) 150(6):935-40. doi: 10.1176/ajp.150.6.935

3. Angst J, Angst F, Gerber-Werder R, Gamma A. Suicide in 406 mood-disorder patients with and without long-term medication: a 40 to 44 years' follow-up. Arch Suicide Res (2005) 9(3):279-300. doi: 10.1080/13811110590929488

4. Paris J, Zweig-Frank H. A 27-year follow-up of patients with borderline personality disorder. Compr Psychiatry (2001) 42(6):482-7. doi: 10.1053/ comp.2001.26271

5. Nordentoft M, Mortensen PB, Pedersen CB. Absolute risk of suicide after first hospital contact in mental disorder. Arch Gen Psychiatry (2011) 68(10):105864. doi: 10.1001/archgenpsychiatry.2011.113

6. Angst J, Gamma A, Benazzi F, Ajdacic V, Eich D, Rossler W. Toward a redefinition of subthreshold bipolarity: epidemiology and proposed criteria for bipolar-II, minor bipolar disorders and hypomania. J Affect Disord (2003) 73 (1-2):133-46. doi: 10.1016/S0165-0327(02)00322-1

7. Angst J, Azorin JM, Bowden CL, Perugi G, Vieta E, Gamma A, et al. Prevalence and characteristics of undiagnosed bipolar disorders in patients with a major depressive episode: the BRIDGE study. Arch Gen Psychiatry (2011) 68(8):791-8. doi: 10.1001/archgenpsychiatry.2011.87

8. Holma KM, Haukka J, Suominen K, Valtonen HM, Mantere O, Melartin TK, et al. Differences in incidence of suicide attempts between bipolar I and II disorders and major depressive disorder. Bipolar Disord (2014) 16(6):652-61. doi: 10.1111/bdi.12195

9. Rodante DE, Grendas LN, Puppo S, Vidjen P, Portela A, Rojas SM, et al. Predictors of short- and long-term recurrence of suicidal behavior in borderline personality disorder. Acta Psychiatr Scand (2019) 140(2):158-68. doi: 10.1111/acps.13058

10. Klonsky ED, May AM. Differentiating suicide attempters from suicide ideators: a critical frontier for suicidology research. Suicide Life Threat Behav (2014) 44(1):1-5. doi: 10.1111/sltb.12068

11. Baldessarini RJ, Tondo L, Pinna M, Nunez N, Vazquez GH. Suicidal risk factors in major affective disorders. Br J Psychiatry: J Ment Sci (2019) 215(4): 1-6. doi: 10.1192/bjp.2019.167

12. Aaltonen KI, Rosenstrom T, Baryshnikov I, Karpov B, Melartin T, Suominen $\mathrm{K}$, et al. Mediating role of borderline personality disorder traits in the effects of childhood maltreatment on suicidal behaviour among mood disorder patients. Eur Psychiatry: J Assoc Eur Psychiatrists (2017) 44:53-60. doi: 10.1016/j.eurpsy.2017.03.011

13. Hawton K, Casanas ICC, Haw C, Saunders K. Risk factors for suicide in individuals with depression: a systematic review. J Affect Disord (2013) 147(13):17-28. doi: 10.1016/j.jad.2013.01.004

14. Schaffer A, Isometsa ET, Tondo L, HM D, Turecki G, Reis C, et al. International Society for Bipolar Disorders Task Force on Suicide: metaanalyses and meta-regression of correlates of suicide attempts and suicide deaths in bipolar disorder. Bipolar Disord (2015) 17(1):1-16. doi: 10.1111/ bdi. 12271

15. Isometsa E. Suicidal behaviour in mood disorders-who, when, and why? Can J Psychiatry (2014) 59(3):120-30. doi: 10.1177/070674371405900303

16. Holma KM, Melartin TK, Haukka J, Holma IA, Sokero TP, Isometsa ET. Incidence and predictors of suicide attempts in DSM-IV major depressive disorder: a five-year prospective study. Am J Psychiatry (2010) 167(7):801-8. doi: 10.1176/appi.ajp.2010.09050627

\section{SUPPLEMENTARY MATERIAL}

The Supplementary Material for this article can be found online at: https://www.frontiersin.org/articles/10.3389/fpsyt.2020. 00290/full\#supplementary-material

17. Pallaskorpi S, Suominen K, Ketokivi M, Valtonen H, Arvilommi P, Mantere $\mathrm{O}$, et al. Incidence and predictors of suicide attempts in bipolar I and II disorders: A 5-year follow-up study. Bipolar Disord (2017) 19(1):13-22. doi: 10.1111/bdi. 12464

18. Oquendo MA, Currier D, Mann JJ. Prospective studies of suicidal behavior in major depressive and bipolar disorders: what is the evidence for predictive risk factors? Acta Psychiatr Scand (2006) 114(3):151-8. doi: 10.1111/j.16000447.2006.00829.x

19. Jylha P, Rosenstrom T, Mantere O, Suominen K, Melartin T, Vuorilehto M, et al. Personality disorders and suicide attempts in unipolar and bipolar mood disorders. J Affect Disord (2016) 190:632-9. doi: 10.1016/j.jad.2015.11.006

20. Popovic D, Vieta E, Azorin JM, Angst J, Bowden CL, Mosolov S, et al. Suicide attempts in major depressive episode: evidence from the BRIDGE-II-Mix study. Bipolar Disord (2015) 17(7):795-803. doi: 10.1111/bdi.12338

21. Yen S, Shea MT, Pagano M, Sanislow CA, Grilo CM, McGlashan TH, et al. Axis I and axis II disorders as predictors of prospective suicide attempts: findings from the collaborative longitudinal personality disorders study. $J$ Abnormal Psychol (2003) 112(3):375-81. doi: 10.1037/0021-843X.112.3.375

22. Yen S, Shea MT, Sanislow CA, Grilo CM, Skodol AE, Gunderson JG, et al. Borderline personality disorder criteria associated with prospectively observed suicidal behavior. Am J Psychiatry (2004) 161(7):1296-8. doi: 10.1176/ appi.ajp.161.7.1296

23. Sher L, Fisher AM, Kelliher CH, Penner JD, Goodman M, Koenigsberg HW, et al. Clinical features and psychiatric comorbidities of borderline personality disorder patients with versus without a history of suicide attempt. Psychiatry Res (2016) 246:261-6. doi: 10.1016/j.psychres.2016.10.003

24. Soloff PH, Chiappetta L. Suicidal Behavior and Psychosocial Outcome in Borderline Personality Disorder at 8-Year Follow-Up. J Pers Disord (2017) 31 (6):774-89. doi: 10.1521/pedi_2017_31_280

25. Posner K, Brown GK, Stanley B, Brent DA, Yershova KV, Oquendo MA, et al. The Columbia-Suicide Severity Rating Scale: initial validity and internal consistency findings from three multisite studies with adolescents and adults. Am J Psychiatry (2011) 168(12):1266-77. doi: 10.1176/ appi.ajp.2011.10111704

26. First MB, Spitzer RL, Gibbon M, Williams JBW. Structured Clinical Interview for DSM-IV-TR Axis I Disorders, Research Version, Non-patient Edition. (SCID-I/NP). New York, NY: Biometrics Research, New York State Psychiatric Institute; (2002).

27. First MB, Gibbon M, Spitzer RL, Williams JBW, Benjamin LS. Structured Clinical Interview for DSM-IV Axis II Personality Disorders, (SCID-II). Washington, D.C.: American Psychiatric Press, Inc. (1997).

28. Montgomery SA, Asberg M. A new depression scale designed to be sensitive to change. Br J Psychiatry (1979) 134:382-9. doi: 10.1192/bjp.134.4.382

29. Young RC, Biggs JT, Ziegler VE, Meyer DA. A rating scale for mania: reliability, validity and sensitivity. Br J Psychiatry (1978) 133:429-35. doi: 10.1192/bjp.133.5.429

30. Arntz A, van den Hoorn M, Cornelis J, Verheul R, van den Bosch WM, de Bie AJ. Reliability and validity of the borderline personality disorder severity index. J Pers Disord (2003) 17(1):45-59. doi: 10.1521/pedi.17.1.45.24053

31. Angst J, Gamma A, Benazzi F, Ajdacic V, Eich D, Rössler W. Toward a redefinition of subthreshold bipolarity: epidemiology and proposed criteria for bipolar-II, minor bipolar disorders and hypomania. Validating Biopolar Spectr (2003) 73(1-2):133-46. doi: 10.1016/S0165-0327(02)00322-1

32. Goldman HH, Skodol AE, Lave TR. Revising axis V for DSM-IV: a review of measures of social functioning. Am J Psychiatry (1992) 149(9):1148-56. doi: 10.1176/ajp.149.9.1148

33. Beck AT, S RA, Brown GK. Manual for the Beck Depression Inventory-II. San Antonio, TX: Psychological Corporation; (1996). 
34. Kroenke K, Spitzer RL, Williams JB. The PHQ-9: validity of a brief depression severity measure. J Gen Intern Med (2001) 16(9):606-13. doi: 10.1046/j.15251497.2001.016009606.x

35. Campbell-Sills L, Norman SB, Craske MG, Sullivan G, Lang AJ, Chavira DA, et al. Validation of a brief measure of anxiety-related severity and impairment: the Overall Anxiety Severity and Impairment Scale (OASIS). J Affect Disord (2009) 112(1-3):92-101. doi: 10.1016/j.jad.2008.03.014

36. Zanarini MC, Vujanovic AA, Parachini EA, Boulanger JL, Frankenburg FR, Hennen J. A screening measure for BPD: the McLean Screening Instrument for Borderline Personality Disorder (MSI-BPD). J Pers Disord (2003) 17 (6):568-73. doi: 10.1521/pedi.17.6.568.25355

37. Blumenthal JA, Burg MM, Barefoot J, Williams RB, Haney T, Zimet G. Social support, type A behavior, and coronary artery disease. Psychosom Med (1987) 49(4):331-40. doi: 10.1097/00006842-198707000-00002

38. Beck A. BHS, Beck hopelessness scale : manual. San Antonio, Tex: Psychological Corp (1988).

39. Aaltonen K, Naatanen P, Heikkinen M, Koivisto M, Baryshnikov I, Karpov B, et al. Differences and similarities of risk factors for suicidal ideation and attempts among patients with depressive or bipolar disorders. J Affect Disord (2016) 193:318-30. doi: 10.1016/j.jad.2015.12.033

40. Saveanu R, Etkin A, Duchemin AM, Goldstein-Piekarski A, Gyurak A, Debattista C, et al. The international Study to Predict Optimized Treatment in Depression (iSPOT-D): outcomes from the acute phase of antidepressant treatment. $J$ Psychiatr Res (2015) 61:1-12. doi: 10.1016/j.jpsychires.2014.12.018

41. Trivedi MH, Rush AJ, Wisniewski SR, Nierenberg AA, Warden D, Ritz L, et al. Evaluation of outcomes with citalopram for depression using measurementbased care in $\mathrm{STAR}^{\star} \mathrm{D}$ : implications for clinical practice. Am J Psychiatry (2006) 163(1):28-40. doi: 10.1176/appi.ajp.163.1.28

42. Sokero TP, Melartin TK, Rytsala HJ, Leskela US, Lestela-Mielonen PS, Isometsa ET. Suicidal ideation and attempts among psychiatric patients with major depressive disorder. J Clin Psychiatry (2003) 64(9):1094-100. doi: 10.4088/JCP.v64n0916
43. Marangell LB, Bauer MS, Dennehy EB, Wisniewski SR, Allen MH, Miklowitz DJ, et al. Prospective predictors of suicide and suicide attempts in 1,556 patients with bipolar disorders followed for up to 2 years. Bipolar Disord (2006) 8(5 Pt 2):566-75. doi: 10.1111/j.1399-5618.2006.00369.x

44. Valtonen H, Suominen K, Mantere O, Leppamaki S, Arvilommi P, Isometsa ET. Suicidal ideation and attempts in bipolar I and II disorders. J Clin Psychiatry (2005) 66(11):1456-62. doi: 10.4088/JCP.v66n1116

45. Vuorilehto M, Valtonen HM, Melartin T, Sokero P, Suominen K, Isometsa ET. Method of assessment determines prevalence of suicidal ideation among patients with depression. Eur Psychiatry: J Assoc Eur Psychiatrists (2014) 29 (6):338-44. doi: 10.1016/j.eurpsy.2013.08.005

46. Vita A, De Peri L, Sacchetti E. Antipsychotics, antidepressants, anticonvulsants, and placebo on the symptom dimensions of borderline personality disorder: a meta-analysis of randomized controlled and openlabel trials. J Clin Psychopharmacol (2011) 31:613-24. doi: 10.1097/ JCP.0b013e31822c1636

47. Cristea IA, Gentili C, Cotet CD, Palomba D, Barbui C, Cuijpers P. Efficacy of psychotherapies for borderline personality disorder: a systematic review and meta-analysis. JAMA Psychiatry (2017) 74:319-28. doi: 10.1001/ jamapsychiatry.2016.4287

Conflict of Interest: The authors declare that the research was conducted in the absence of any commercial or financial relationships that could be construed as a potential conflict of interest.

Copyright (c) 2020 Söderholm, Socada, Rosenström, Ekelund and Isometsä. This is an open-access article distributed under the terms of the Creative Commons Attribution License (CC BY). The use, distribution or reproduction in other forums is permitted, provided the original author(s) and the copyright owner(s) are credited and that the original publication in this journal is cited, in accordance with accepted academic practice. No use, distribution or reproduction is permitted which does not comply with these terms. 